\title{
A theory of nonmonotonic rule systems I
}

\author{
W. Marek, ${ }^{1}$ A. Nerode ${ }^{2}$ and J. Remmel ${ }^{3}$
}

\section{Contents}

1 Introduction 2

2 Monotonic formal systems $\quad 14$

3 Nonmonotonic formal systems $\quad 15$

4 Examples and Applications in Logic, Logic Programming, and Commonsense Reasoning $\quad 26$

4.1 Classical Implicational Propositional Logic . . . . . . . . . . . . . . . 26

4.2 Other Classical Systems . . . . . . . . . . . . . . . . 27

4.3 Ordinary Clausal Logic Programming . . . . . . . . . . . . . . . . . 27

4.4 Propositional Logic Programming, monotonic rules case . . . . . . . . 28

4.5 Default logic . . . . . . . . . . . . . . . . . . . . . . . . . 29

4.6 Propositional logic programming, general case . . . . . . . . . . . 30

4.7 Logic programming with classical negation . . . . . . . . . . . . 31

5 Representation of Combinatorial and Algebraic Problems as Structures associated with Rule Systems $\quad 34$

\footnotetext{
${ }^{1}$ Department of Computer Science, University Kentucky, Lexington, KY 40506-0027. Currently in Mathematical Sciences Institute at Cornell University. Work partially supported by NSF grant RII-8610671 and Kentucky EPSCoR program and ARO contract DAAL03-89-K-0124.

${ }^{2}$ Mathematical Sciences Institute, Cornell University, Ithaca, NY 14853. Work partially supported by NSF grant DMS-8902797 and ARO contract DAAG629-85-C-0018.

${ }^{3}$ Department of Mathematics, University of California at San Diego, La Jolla, CA 92903. Work partially supported by NSF grant DMS-8702473.
} 
5.1 The Marriage Problem . . . . . . . . . . . . . . . . . 34

5.2 Proper $k$-colorings of graphs . . . . . . . . . . . . . . . . 38

5.3 Chain Covers of Partially Ordered Sets . . . . . . . . . . . . . . 40

5.4 Maximal Ideals in a Boolean Algebra . . . . . . . . . . . . . . . . . . 42

5.5 Complementary Subspaces of Vector Spaces . . . . . . . . . . . . . 44

5.6 Prime ideals in a commutative ring $\mathcal{R}$, the radical and skeptical reasoning 47

6 Conclusions and the Contents of Part II 50

\section{Introduction}

In mathematics, a consequence drawn by a deduction from a set of premises can also be drawn by the same deduction from any larger set of premises. The deduction remains a deduction no matter how the axioms are increased. This is monotonic reasoning, much imitated in other, less certain, disciplines. The very nature of monotonic reasoning makes mathematical proofs permanent, independent of new information. Thus it has been since Euclid and Aristotle. Theorems with complete proofs are never withdrawn due to later knowledge. It is little exaggeration to say that mathematicians never reject the completed proofs of their predecessors, except to complain about their constructivity.

Mathematicians build directly on the works of their forebearers stretching back two and a half millenia to Euclid. Our current mathematical reasoning is merely a fleshed out version of Euclid's. Monotonic reasoning marks theoretical mathematics as a discipline. The traditional systems of mathematical logic are monotonic since they simply reflect mathematical usage. Tarski [1956] described a calculus of de- 
ductive systems and captured in a simple way the general concept of a monotonic formal system. His formulation includes all logics traditionally studied: intuitionistic, modal, and classical. He did not qualify his definition, as we do, with the adjective "monotone," because there were no other systems studied at that time.

Minsky [1975] suggested that there is another sort of reasoning which is not monotonic. This is reasoning in which we deduce a statement based on the absence of any evidence against the statement. Such a statement is in the category of beliefs rather than in the category of truths. Modern science offers statistics as a tool for establishing provisional beliefs, but in many instances we have no basis for applying statistics, due to a lack of governing distributions or samples for the problem at hand.

What role does belief play in our affairs? Often we must make sharp "yes or no" decisions between alternative actions. There may be no deductive or statistical base which justifies our choice, or there may be such a base but we may not be able to wait for missing information; it may never materialize anyway. Often all we have as a basis for decision is surmise, that is, deductions from beliefs as well as truths and statistically derived statements. These beliefs are often accepted, and used, as premises for deduction and choice of action due to an unquantified lack of evidence against them.

A philosopher's much-quoted example is about Tweety. We observe only birds that can fly, and accept the belief that all birds can fly from the absence of evidence for the existence of non-flying birds. We are told that Tweety is a bird, and conclude that Tweety can fly using our belief as premise. Later, we observe that Tweety is 
a pet ostrich and clearly can't fly. We reject our previous belief set and conclusions as a basis for decision making, and are forced to choose a new belief set. The new set of beliefs may also include equally uncertain statements, accepted due to a lack of evidence against them. But we blithely draw consequences from the new belief set and make decisions on that basis till contrary evidence on some accepted belief is garnered, at which time we again have to acquire a new set of beliefs.

This has happened in the history of practically every subject except mathematics. The principles of physics, or biology, have been changed with every scientific revolution, even though unreflective practitioners of each age think that final principles have been found. For mathematics, the Dutch mathematician and philosopher L.E.J. Brouwer would have argued that the belief in theorems established by "nonconstructive methods" was unjustified, and that a new belief set based on constructive principles should be adopted in its place. Other mainstream mathematicians, such as Hilbert, did not agree with this position. Some philosophers of mathematics living now would argue that, even within classical mathematics, the independence proofs for propositions of set theory, such as the continuum hypothesis or the axiom of choice, indicate there are several incompatible axiomatic systems which, as belief sets, could be the foundation of mathematics.

One can envisage making up nonmonotone logics describing the mathematical nature of belief. The exact result depends on the definition chosen for "lack of evidence against". McCarthy [1980], initiated the study of nonmonotonicity with his notion of circumscription. With all relation symbols but one, $\bar{R}$, of a model (the world we 
are discussing) held fixed, and given axioms $\varphi(\bar{R})$ relating that $\bar{R}$ to the other (fixed) relations of the model, the belief should be that, lacking further evidence to the contrary, $\bar{R}$ denotes the least relation $R$, if any, satisfying $\varphi(R)$. If further evidence in the form of an additional axiom $\psi(\bar{R})$ becomes available, then we should believe that $\bar{R}$ denotes the least $R$ satisfying $(\varphi \wedge \psi)(R)$, if any, instead, in a changed belief set.

There are now many different nonmonotonic system, abstracted from different questions in computer science and AI. Among the other systems that have been studied are

Theory of multiple believers of Hintikka, [1962].

Truth Maintenance systems of Doyle, [1979]

Default logic of Reiter, [1980]

Autoepistemic logic of Moore, [1985]

Theory of individual and common knowledge and belief of Halpern and Moses [1984]

Logic programming with negation as failure [Apt, 1988].

This, by no means, exhausts the list. What issues in artificial intelligence or computer science motivate these systems?

Suppose that we build a robot in a "blocks world" to navigate in a room, avoid obstacles and perform simple tasks, such as crossing the room with variable obstacles. 
We want the robot to learn principles from experience as to how to cross the room. At any given point, one may imagine that the robot should have a consistent deductively closed set of beliefs which are the current basis for its actions, including such provisional beliefs as "I can always traverse the left edge of the room since there has never been anything in the way there". But when such a principle is contradicted by new obstacles, the robot has to choose another belief set. So an important problem is to define what a belief set is and how to compute them and how to update them based on new evidence. Moore's autoepistemic logic [1985], is really a first try at this problem, mostly for propositional logic.

In computers, the operating system and program obey rules which compute how to change state. In the absence of exceptional behaviour, such as an error condition or failure to access resources, there is a system of decision rules (beliefs) computing how to change the state of the machine in this "normal behavior", or "default" case. But when an exceptional behavior happens, we are thrown to a different set of decision rules for change of state, a different set of "beliefs". One wants to be able to deduce what is true of the machine in states when it is in a particular such "belief set". A logic for dealing with one such belief set at a time is Reiter's default logic.

In databases, facts and rules are stored as entries (the PROLOG model). Often also the database computes and stores conclusions, such as summary statistics or rules or tables computed from the database. These act as a deductive base for the set of current beliefs. When we query the database, we are asking for consequences of this belief set. When we update the database, all old entries that have changed have to 
be replaced and every consequence that uses these entries has to be recomputed and changed too. This is the process of replacing an old belief set by a new one. One often makes decisions on the basis of the absence of information in the database as well. A logic appropriate for describing a single such belief set is Doyle's truth-maintenance system [1979]. See also de Kleer [1986]. Also stable models for logic programming with negation as failure ([Gelfond and Lifschitz, 1988]) arise in this way.

We expressed these examples informally in terms of the anthropomorphic notion of belief so as to bring out their common features. The actual nonmonotonic logics have much in common, and a number of translations between them have been proposed ([Konolige, 1988], [Gelfond and Przymusińska, 1986],[Gelfond and Przymusińska, 1989], [Reinfrank and Dressler, 1989],[Marek and Truszczyński, 1989]). They have been investigated principally for propositional logic. Predicate versions suitable for actual applications are, up to now, pretty minimal.

Study of monotonic rule systems can be traced to the work of Post on "production systems" and to work of Tarski on the abstract properties of consequence relations for classical logic systems. The investigation of the nonmonotonic component is of much more recent nature and seems to appear first in the work of Reiter on default logic. Reiter's investigations involved finding a natural extension of classical logic which allows one to handle the negative information.

Independently Clark, and subsequently Apt, Blair and Walker, and also (extending their work) Gelfond and Lifschitz, studied negation as failure in logic programming. It has turned out that these investigations are in a common direction. $\mathrm{Mu}-$ 
tual relationships were uncovered by Bidoit and Froidevaux, [1988], and Marek and Truszczynski, [1989b], who exhibited the precise nature of the connection between logic programming and default logic. The reevaluation of default extensions in terms of "context-dependent proofs" by Marek and Truszczynski, which has its roots in the Apt, Blair and Walker's ([1987]) "elementary interpreter", for which it may serve as a clarifying definition, is a point of departure for the investigations of this paper. Here, drawing on all the research mentioned above for inspiration, we present a coherent unified theory of nonmonotonic formal systems.

At the level of abstraction we achieve, we are finally able to see that nonmonotone systems pervade ordinary mathematical practice. There is no sign of any realization of the existence of such mathematical examples in the previous nonmonotonic logic literature. Perhaps these connections can only be seen by having a common abstract notion. What this commonality does for us is to make available known mathematical techniques from other areas of conventional mathematics for constructing and classifying belief sets (extensions) and, simultaneously, to make evident a common thread among disparate parts of mathematics and disparate nonmonotonic systems from artificial intelligence and computer science.

On the level of Mathematical Philosophy there is a connection worth stating as well. Non-monotone reasoning takes place during the process of discovery of mathematical theorems, when one posits temporarily some proposition on the basis of no evidence against it, and explores the consequences of such a belief until new mathematical facts force their abandonment. These nonmonotone belief sets have their 
traces eradicated when final belief sets are achieved and demonstrative proofs are finished and published. The only hint of provisional belief sets left in mathematical papers is in the motivational remarks explaining what obstacles were overcome and by what changes in viewpoint the proof was achieved.

Here is the main definition. A nonmonotone rule system consists of a set $U$ and a set of triples $(\alpha, \beta, \gamma)$ called rules. Here $\alpha=\left(\alpha_{1}, \ldots, \alpha_{n}\right)$ is a finite sequence of elements of $U$, called premises, and $\beta=\left(\beta_{1}, \ldots, \beta_{k}\right)$ is a finite sequence of elements from $U$, called restraints, and $\gamma$ is an element of $U$. This is written, generalizing a notation of default logic, as

$$
\frac{\alpha_{1} \ldots, \alpha_{n}: \beta_{1}, \ldots, \beta_{k}}{\gamma}
$$

The informal reading is: From $\alpha_{1}, \ldots, \alpha_{n}$ being established, and $\beta_{1}, \ldots, \beta_{k}$ not being established now or ever, conclude $\gamma$. You may substitute "computed" for "established" for an informal reading in many applications. A subset $S$ of $U$ is called deductively closed if for every rule of the system, whenever $\alpha_{1}, \ldots, \alpha_{n}$ are in $S$ and $\beta_{1}, \ldots, \beta_{k}$ are not in $S$, then $\gamma$ is in $S$. There are no variables here, these are not schema, this version is not the one appropriate for nonmonotone predicate logics. Nonmonotonic predicate logic cannot be exposited in a few lines and we defer that to a later paper.

The intersection of all deductively closed sets containing a set $I$ is generally not deductively closed. But the intersection of a descending chain of deductively closed sets is deductively closed, and I may be contained in many minimal deductively closed sets over $I$. In the context of nonmonotone logic the intersection of all deductively 
closed sets containing $I$ is a (non-deductively closed) set, called the set of secure consequences of $I$. These are the propositions that a "skeptical reasoner" would take as beliefs based on $I$. The most important notion of contemporary nonmonotonic logic is that of extension. For a fixed subset $S$ of $U$, one defines (finite) derivations from $I$, where all restraints encountered are outside $S$, and all premises encountered are either conclusions of previous rules or in $I$. This defines the set $C_{S}(I)$ of $S$-consequences of I. Extensions are those $S$ such that $S=C_{S}(I)$. Extensions are minimal deductively closed sets containing I, but not conversely. Extensions represent the "deductively closed belief sets" that contain $I$. In these sets, if the negative restraints are all obeyed, we are reduced to monotone reasoning. See Section 3 for the exact definition.

These simple definitions capture the common content of the several theories of nonmonotonicity listed above, and of many mathematical theories as well. For example, the set of all marriages of the marriage problem can be formulated as exactly the set of all extensions in a nonmonotone rule system; similarly for the set of all $k$-colorings of graphs, the set of chain covers of partial orders, the Stone space of all maximal ideals in a Boolean algebra, etc. Similarly, for a commutative ring with unit there is a nonmonotone rule system such that the deductively closed sets are the prime ideals, the McCoy radical (the set of nilpotents) is the set of secured consequences of $\{0\}$, etc. There are similar nonmonotonic systems associated with virtually every algebraic system for which radicals of some sort have been defined and characterized. These mathematical examples have suggested a whole new set of techniques for finding extensions because of the availability of algorithms already investigated in the 
mathematical literature on one or another of these problems, not previously known to be relevant to nonmonotonic logic in the artificial intelligence community. They do not arise from logic, but really from operations research. Finally, in recursion theory, priority constructions can be construed as nonmonotone systems, sets constructed by the priority argument as extensions. These ideas give many constructions of recursively enumerable extensions.

We spend a lot of effort in both this and subsequent papers to answer the following question. Exactly how complicated is the set of extensions of a recursively specified nonmonotonic system, and what is its structure? This is the analogue of the classical logic question, how complicated is the set of complete theories containing a recursively enumerable theory, and what is its structure? In classical logic, this leads to analyzing the character of the set of maximal ideals containing a given recursively enumerable ideal in a recursively presented free Boolean Algebra, a subject in which two of the authors have a lot of experience (see [Remmel, 1989], [Nerode and Remmel, 1985]). The simplest case covering many nonmonotonic systems arising from mathematics is that of "highly recursive" nonmonotone rule systems. There it turns out that extensions can, up to a one-to-one recursive map, be exactly any bounded $\Pi_{1}^{0}$ class of sets of natural numbers. So even in this case the computational problems are of the same level of difficulty as (say) solving "marriage problem" for highly recursive societies, or finding orderings of recursively presented formally real field ([Metakides and Nerode, 1977]), or finding an abcissa between 0 and 1 where a given recursive continuous function on $[0,1]$ takes a maximum value ([Huang and Nerode, 1985]). 
This recursion-theoretic methodology can also be refined to give complexity-theoretic results on the same problems about extensions, as has been done in algebra by Nerode and Remmel in [1987], [1989], and [1990]. Since this is a more delicate matter than the recursion theory, these developments are deferred again to a later paper.

Next, we turn to investigations of the semantics of nonmonotonic rule systems. The fundamental common semantics we have found comes from $L_{\omega_{1} \omega}$, and generalizes the Clark completion of logic programming. It is perfectly general, and gives systematic semantics and completeness for all the nonmonotonic logics discussed above. Such uniform semantics are new. Some of the subjects never before had a decent semantics. We find semantical representations of extensions, weak extensions and deductively closed sets. This representation requires the creation of an additional infinitary language $\mathcal{L}_{S}$ which properly encodes not only rules as "first order objects", but also additional (infinitary) objects which characterize the class of intended structures be they extensions, weak extensions, deductively closed sets, etc. The previously established characterization of default logic, in terms of nonmonotonic rule systems, provides us with a semantics for default logic. This semantics, in opposition to the attempt of Etherington, satisfies Tarski's conditions. That is, it allows us to introduce for defaults (virtual) negations, conjunctions, etc, and also a natural entailment relation. Computer scientists have a prejudice against $L_{\omega_{1} \omega}$ since on the surface it is removed from computation. But this is only on the surface - well-founded relations of small recursive ordinal height are the "correct" basis for our algorithms for computing extensions and represent $L_{\omega_{1} \omega}$ deductions - so perhaps the prejudice against 
$L_{\omega_{1} \omega}$ should be reconsidered.

Finally, we turn to the issue of computing extensions, weak extensions, and sets closed under rules. We provide algorithms for testing whether a given subset of the universe $U$ is an extension, weak extension, etc. Since we have previously provided a reduction algorithm to the monotonic case, an extension of the Truszczynski variant of the Gelfond-Lifschitz procedure for testing stability, we are able to use an analytical tableau method to compute the least fixpoint of a monotonic operator.

This short summary indicates that there is a great wealth of problems and results which naturally arise from nonmonotonic rule systems. Our study delineates the role of deduction schemata. This, in turn, connects our work naturally with studies of inductive definability. Our work indicates that logic programming is less related to predicate calculus, than to inductive definability. This is a paradigm different from Kowalski's. We do not claim that this is the only "correct" position, but we do claim that it leads to a new direction for research.

The predicate logic case is not treated in this paper. It will come out from a schematic version of the theory of this paper analogous to Post production systems. Along with $U$, the set of all strings over an alphabet, there are typed "metavariables" ranging over specific subsets of $U$ called "types". There are "metastrings" built from the alphabet of $U$ and string variables. Rules are of the same form as before, but use metastrings instead of strings. This point of view gives rise not only to a general theory, but also gives outright syntax, semantics, and completeness for new predicate versions of all the logics mentioned above. It also gives nonmonotone classical, or 
intuitionistic, or modal predicate and propositional logics.

\section{Monotonic formal systems}

Tarski [1956] characterized monotonic formal systems by means of monotonic rules of inference. Such systems include intuitionistic logic, classical logics, modal logics, and many others. Suppose that a nonempty set $U$ is given. In a particular application $U$ may be the collection of all statements or all formulas, or all legal strings of a formal system, or of all atomic statements as in logic programming.

A monotonic rule of inference is a tuple $r=\left\langle P, \varphi>\right.$, where $\left.P=<\alpha_{1}, \ldots, \alpha_{n}\right\rangle$ is a finite list of objects from $U$, and $\varphi$ is an element of $U$. Such a rule $r$ is usually written in the suggestive form

$$
r=\frac{\alpha_{1}, \ldots, \alpha_{n}}{\varphi}
$$

We call $\alpha_{1}, \ldots, \alpha_{n}$ the premises of $r$ and $\varphi$ the conclusion of $r$.

Definition: (a) A monotonic formal system is a pair $\langle U, M\rangle$, where $U$ is a nonempty set and $M$ is a collection of monotonic rules.

(b) A subset $S \subseteq U$ is called deductively closed over $<U, M>$ if for all rules $r \in M$, $\alpha_{1}, \ldots, \alpha_{n} \in S$ implies $\varphi \in S$.

The collection $D=D(U, M)$ of deductively closed sets has the following properties:

(1) $U \in D$

(2) $D$ is closed under arbitrary intersections. 
Consequently, for every $I \subseteq U$ there is the least set $T(I)$ such that $I \subseteq T(I)$ and $T(I)$ is deductively closed. The operation $T\left(=T_{U, M}\right)$ is monotone, that is, if $I \subseteq J$ then $T(I) \subseteq T(J)$. Moreover,

(3) $T(I)=\bigcup\{T(J): J \subseteq I \&|J|<\omega\}$.

(Where $\omega$ is the cardinality of of the set of natural numbers.)

Property (3) reflects the finitary nature of deductive closure and is closely associated with the definition of a deduction.

An axiom is a rule without premises, that is, with the list $P$ empty.

A deduction of an object $\varphi \in U$ from $I \subseteq U$ is a finite sequence $\left\langle\varphi_{1}, \ldots, \varphi_{m}\right\rangle$ such that $\varphi_{m}=\varphi$, and for all $i \leq m, \varphi_{i} \in I$, or $\varphi_{i}$ is an axiom, or $\varphi_{i}$ is the conclusion of a rule $r \in M$ such that premises of $r$ are included in $\left\{\varphi_{1}, \ldots, \varphi_{i-1}\right\}$. Then $S(I)$ consists of all elements of $U$ that possess a deduction from $I$.

Tarski made the easy observation that if a collection $D$ of subsets of $U$ possesses properties (1), (2) and (3) above, then there is a collection of monotone rules $M$ such that $D$ is the set of all deductively closed sets in $\langle U, M\rangle$.

An abstract treatment of monotonic logic programming schemes and general methods of processing queries is discussed in [Blair, Brown, and Subrahmanian, 1989].

\section{$3 \quad$ Nonmonotonic formal systems}

Inspired by Reiter [1980], and Apt [1988], we introduce the notion of a nonmonotonic formal system $\langle U, N\rangle$. A nonmonotonic rule of inference is a triple $\langle P, G, \varphi\rangle$, 
where $P=\left\{\alpha_{1}, \ldots, \alpha_{n}\right\}, G=\left\{\beta_{1}, \ldots, \beta_{m}\right\}$ are finite lists of objects from $U$ and $\varphi \in U$. Each such rule is written in form

$$
r=\frac{\alpha_{1}, \ldots, \alpha_{n}: \beta_{1}, \ldots, \beta_{m}}{\varphi}
$$

Here $\left\{\alpha_{1}, \ldots, \alpha_{n}\right\}$ are called the premises of rule $r,\left\{\beta_{1}, \ldots, \beta_{m}\right\}$ are called the $r e$ straints of rule $r$.

Either $P$, or $G$, or both may be empty. If $P=G=\emptyset$ then the rule $r$ is called an axiom.

A nonmonotonic formal system is a pair $\langle U, N\rangle$, where $U$ is a non-empty set and $N$ is a set of nonmonotonic rules.

Each monotonic formal system can be identified with the nonmonotonic system in which every monotonic rule is given an empty set of restraints.

A subset $S \subseteq U$ is called deductively closed if for every rule of $N$, if all premises $\alpha_{1}, \ldots, \alpha_{n}$ are in $S$ and all restraints $\beta_{1}, \ldots, \beta_{m}$ are not in $S$ then the conclusion $\varphi$ belongs to $S$.

In nonmonotonic systems, deductively closed sets are not generally closed under arbitrary intersections as in the monotone case. Tarski's axioms do not generally hold. But deductively closed sets are closed under intersections of descending chains. Since $U$ is deductively closed, by the Kuratowski-Zorn Lemma, any $I \subseteq U$ is contained in at least one minimal deductively closed set. The intersection of all the deductively closed sets containing $I$ is called the set of secured consequences of $I$. This set is also the intersection of all minimal deductively closed sets containing $I$. Deductively 
closed sets are thought of as representing possible "points of view". The intersection of all deductively closed sets containing $I$ represents the common information present in all such "points of view", containing $I$. (Generally in the literature, if we assign to a given $I$ a collection $\mathcal{M}$ of subsets of $U$, then assigning to $I$ the intersection of $\mathcal{M}$ is called the skeptical reasoning associated with $\mathcal{M}$ and $I$.)

Example 3.1 Let $U=\{\alpha, \beta, \gamma\}$.

(a) Consider $U$ with $N_{1}=\left\{\frac{:}{\alpha}, \frac{\alpha: \beta}{\beta}\right\}$. There is only one minimal deductively closed set $S=\{\alpha, \beta\}$. Then $\{\alpha, \beta\}$ is the set of secured consequences of $<U, N_{1}>$.

(b) Consider $U$ with $N_{2}=\left\{\frac{\dot{\alpha}}{\alpha}, \frac{\alpha: \beta}{\gamma}, \frac{\alpha: \gamma}{\beta}\right\}$, then there are two minimal deductively closed sets, $S_{1}=\{\alpha, \beta\}, S_{2}=\{\alpha, \gamma\} .\{\alpha\}$ is the set of secured consequences of $<U, N_{2}>$.

Example 3.1, (b) shows that the set of all secured consequences is not, in general, deductively closed in the nonmonotone case.

Let $\mathcal{P}(U)$ be collection of all subsets of $U$. Let $\mathcal{X} \subseteq \mathcal{P}(U)$ be given. Call a set $X$ a hitset for $\mathcal{X}$ if for all $Y \in \mathcal{X}, X \cap Y \neq \emptyset$. For each rule $r=\frac{\alpha_{1}, \ldots, \alpha_{n}: \beta_{1}, \ldots, \beta_{m}}{\varphi}$, define $Y_{r}=\left\{\beta_{1}, \ldots, \beta_{m}, \varphi\right\}$. For $<U, N>$, let $\mathcal{X}_{U, N}=\left\{Y_{r}: r \in N\right\}$. The following is a sufficient condition for $X$ to be a deductively closed set in $\langle U, N\rangle$.

Proposition 3.1 If $X$ is a hit set for $\mathcal{X}_{U, N}$, then $X$ is deductively closed in $\langle U, N\rangle$.

The converse to Proposition 3.1 does not hold. If $U=\{\alpha, \beta, \gamma\}, N=\left\{\frac{\alpha: \beta}{\gamma}\right\}$, then $\emptyset$ is deductively closed for $\langle U, N\rangle$, but this is not a hit set for $\mathcal{X}_{U, N}$. 
When $N$ is finite, it is easy to give an algorithm based on Propositiom 3.1 for finding all minimal deductively closed sets. This algorithm is a variant of a "brute force" algorithm based on enumerating all hit sets.

Given a set $S$ and an $I \subseteq U$, an $S$-deduction of $\varphi$ from $I$ in $\langle U, N\rangle$ is a finite sequence $\left\langle\varphi_{1}, \ldots, \varphi_{k}>\right.$ such that $\varphi_{k}=\varphi$ and, for all $i \leq k$, each $\varphi_{i}$ is in $I$, or is an axiom, or is the conclusion of a rule $r \in N$ such that all the premises of $r$ are included in $\left\{\varphi_{1}, \ldots, \varphi_{i-1}\right\}$ and all restraints of $r$ are in $U \backslash S$ (see [Marek and Truszczyński, 1989], also [Reinfrank and Dressler, 1989]). An $S$-consequence of $I$ is an element of $U$ occurring in some $S$-deduction from $I$. Let $C_{S}(I)$ be the set of all $S$-consequences of $I$ in $\langle U, N\rangle$. $I$ is a subset of $C_{S}(I)$. Note that $S$ enters solely as a restraint on the use of the rules imposed by the restraints in the rules. A single restraint in a rule in $N$ may be in $S$ and therefore prevent the rule from ever being applied in an $S$-deduction from $I$, even though all the premises of that rule occur earlier in the deduction. Thus $S$ contributes no members directly to $C_{S}(I)$, although members of $S$ may turn up in $C_{S}(I)$ by an application of a rule which happens to have its conclusion in $S$. For a fixed $S$, the operator $C_{S}(\cdot)$ is monotonic. That is, if $I \subseteq J$, then $C_{S}(I) \subseteq C_{S}(J)$. Also, $C_{S}\left(C_{S}(I)\right)=C_{S}(I)$.

Generally, $C_{S}(I)$ is not deductively closed in $\langle U, N\rangle$. It is perfectly possible that all the premises of a rule be in $C_{S}(I)$, the restraints of that rule are outside $C_{S}(I)$, but a restraint of that rule be in $S$, preventing the conclusion from being put into $C_{S}(I)$.

Example 3.2U $=\{\alpha, \beta, \gamma\}, N=\left\{\frac{:}{\alpha}, \frac{\alpha: \beta}{\gamma}\right\}, S=\{\beta\}$. Then $C_{S}(\emptyset)=\{\alpha\}$ is not 
deductively closed.

However, the following holds:

Proposition 3.2 If $S \subseteq C_{S}(I)$ then $C_{S}(I)$ is deductively closed.

Proof: If all the premises of a rule $r$ are in $C_{S}(I)$ and all the restraints are outside $C_{S}(I)$, then all the restraints are also outside $S$.

We say that $S \subseteq U$ is grounded in $I$ if $S \subseteq C_{S}(I)$.

We say that $S \subseteq U$ is an extension of $I$ if $C_{S}(I)=S$.

Finally, we say that $S \subseteq U$ is a weak extension of $I$ if $C_{S}(I \cup R)=S$,

where

$R=\left\{\varphi\right.$ : for some $\left.r \in N, r=\frac{\alpha_{1}, \ldots, \alpha_{n}: \beta_{1}, \ldots, \beta_{m}}{\varphi}, \alpha_{1}, \ldots, \alpha_{n} \in S, \beta_{1}, \ldots, \beta_{m} \notin S\right\}$

Thus $S$ is a weak extension if $S$ is generated by $I$ and the conclusions of rules that are applicable. The notion of weak extension is related to Clark's logic program completion and will be investigated below. The notion of groundedness is related to the phenomenon of "reconstruction". $S$ is grounded in $I$ if all elements of $S$ are $S$-deducible from $I$ (remember that $S$ influences only the negative sides of rules). $S$ is an extension of $I$ if two things happen. First, every element of $S$ is deducible from $I$, that is, $S$ is grounded in $I$ (this is an analogue of adequacy property in logical calculi). Second, the converse holds: all the $S$-consequences of $I$ belong to $S$ (this is the analogue of completeness). Thus extensions are analogues for a nonmonotonic systems of the set of all consequences for monotonic systems. Both properties (adequacy and completeness) need to be satisfied - if we want $S$ to be an extension. 
The third concept, weak extension, is a closure property. In the process of constructing $C_{S}(I), S$ is used to generate only negatively as a restraint. But we can relax our requirements and allow deductions that use $S$ also on the positive side. That is, elements of $S$ are not treated as "axioms", but are used to generate objects from $U$ by also testing the positive side of a rule for membership in $S$. This concept is closely related with the fixpoints of the operator $T_{P}$ in logic programming, and Clark's completion, see [Apt, 1988]. We shall prove a similar representation of weak extensions as fixpoints of an operator associated with a nonmonotonic system.

The notion of an extension is related to that of a minimal deductively closed set.

Lemma 3.3 If $S$ is an extension of $I$, then:

(1) $S$ is a minimal deductively closed superset of $I$.

(2) For every $I^{\prime}$ such that $I \subseteq I^{\prime} \subseteq S, C_{S}\left(I^{\prime}\right)=S$.

Proof: (1) Clearly, $S$ contains $I$. The operator $C_{S}$ is anti-monotonic in $S$. That is, $S_{1} \subseteq S_{2}$ implies $C_{S_{2}}(I) \subseteq C_{S_{1}}(I)$. Thus, if $S$ is an extension of $I$ and $S^{\prime} \subseteq S$ is deductively closed, then $S^{\prime} \subseteq S \subseteq C_{S}(I) \subseteq C_{S^{\prime}}(I) \subseteq S^{\prime}$. So $S=S^{\prime}$.

(2) Assume that $I \subseteq I^{\prime} \subseteq S$ and that $C_{S}(I)=S$. Then $C_{S}(I) \subseteq C_{S}\left(I^{\prime}\right) \subseteq$ $C_{S}\left(C_{S}(I)\right) \subseteq C_{S}(I)=S$

Proposition 3.4 The set of extensions of I forms an antichain. That is, if $S_{1}, S_{2}$ are extensions of $I$ and $S_{1} \subseteq S_{2}$, then $S_{1}=S_{2}$.

Proof: $S_{1} \subseteq S_{2} \subseteq C_{S_{2}}(I) \subseteq C_{S_{1}}(I)=S_{1}$ 
Proposition 3.5 An extension of $I$ is a weak extension of $I$.

Proof: Assume that $S$ is an extension of $I$, that is, $S=C_{S}(I)$. We need to prove that $S=C_{S}(I \cup R)$, where $R$ is

$$
\left\{\varphi \text { : for some } r \in N, \quad r=\frac{\alpha_{1}, \ldots, \alpha_{n}: \beta_{1}, \ldots, \beta_{m}}{\varphi}, \alpha_{1}, \ldots, \alpha_{n} \in S, \beta_{1}, \ldots, \beta_{m} \notin S\right\}
$$

Then

$$
S \subseteq C_{S}(I) \subseteq C_{S}(I \cup R) \subseteq C_{S}(S)=S,
$$

So $S=C_{S}(I \cup R)$.

Given $S \subseteq U$, a rule $r$ is called $S$-applicable if all the restraints of $r$ are outside $S$ and all the premises of $r$ are in $S$. We define $N(S)$ to be the collection of all $S$-applicable rules.

With a nonmonotonic system $\mathcal{S}=\langle U, N\rangle$ we associate the operator $T=$ $T_{\mathcal{S}}: \mathcal{P}(U) \rightarrow \mathcal{P}(U)$ defined as follows:

$$
T_{\mathcal{S}}(I)=\left\{\varphi \in U: \exists_{r \in N} r=\frac{\alpha_{1}, \ldots, \alpha_{n}: \beta_{1}, \ldots, \beta_{m}}{\varphi}, \alpha_{1}, \ldots, \alpha_{n} \in I, \beta_{1}, \ldots, \beta_{m} \notin I\right\}
$$

This operator is closely related to the operator $T_{P}$ as considered in logic programming, see [Apt, 1988]. As in the case of general logic programs, the operator $T$ is not monotone in general. But if $\mathcal{S}$ is a monotone system, that is all rules in $N$ have no restraints, then $T$ is monotone. Operator $T$ computes the collection of conclusions of $I$-applicable rules.

The following result generalizes one for logic programming. 
Proposition 3.6 Let $\langle U, N\rangle$ be a nonmonotonic rule system. Let $T$ be its associated operator, and let $S \subseteq U$. Then:

(1) $T(S) \subseteq S$ if and only if $S$ is deductively closed.

(2) $T(S)=S$ if and only if $S$ is a weak extension of $\emptyset$ in $\langle U, N\rangle$.

Proof: (1) restates the definition of deductively closed set.

$(2) \Rightarrow$. We assume that $T(S)=S$ and then prove that $S$ is a weak extension of $\emptyset$ in $<U, N>$. We must show that $S=C_{S}(R)$ where $R$ is the set

$$
\left\{\varphi \text { : for some } r \in N, r=\frac{\alpha_{1}, \ldots, \alpha_{n}: \beta_{1}, \ldots, \beta_{m}}{\varphi}, \alpha_{1}, \ldots, \alpha_{n} \in S, \beta_{1}, \ldots, \beta_{m} \notin S\right\}
$$

(That is the collection of conclusions of $S$-applicable rules). To this end we need to prove two inclusions:

(a) $S \subseteq C_{S}(R)$. But we know that $S=T(S)$, that is, every $\varphi \in S$ is a conclusion of an $S$-applicable rule $r=\frac{\alpha_{1}, \ldots, \alpha_{n}: \beta_{1}, \ldots, \beta_{m}}{\varphi}$, with $\alpha_{1}, \ldots, \alpha_{n} \in S, \beta_{1}, \ldots, \beta_{m} \notin S$. Thus $\varphi \in R \subseteq C_{S}(R)$

(b) $C_{S}(R) \subseteq S$. If $\gamma \in C_{S}(R)$, then there exists an $S$-deduction $d$ from $R$ with the conclusion $\gamma$. We leave the reader to check by induction on the length of such a deduction $d$ that the conclusion of $d$ is in $S$.

$\Leftarrow$. We now assume that $S$ is a weak extension of $\emptyset$ in $\langle U, N\rangle$. That is $C_{S}(R)=S$, where $R$ is the set

$$
\left\{\varphi \text { : for some } r \in N, r=\frac{\alpha_{1}, \ldots, \alpha_{n}: \beta_{1}, \ldots, \beta_{m}}{\varphi}, \alpha_{1}, \ldots, \alpha_{n} \in S, \beta_{1}, \ldots, \beta_{m} \notin S\right\} \text {. }
$$

We prove that $S=T(S)$.

(a) $T(S) \subseteq S$. If $\alpha \in T(S)$ then by definition $\alpha$ is a conclusion of an $S$-applicable 
rule. Thus $\alpha \in R \subseteq C_{S}(R)=S$.

(b) $S \subseteq T(S)$. By our assumption, if $\alpha \in S$ then $\alpha \in C_{S}(R)$. By induction on the length of an $S$-deduction of $\alpha$, we leave the reader to prove that $\alpha$ belongs to $T(S)$.

We get as an immediate corollary

Proposition 3.7 Let $S \subseteq U$. Then $S$ is a weak extension of $\emptyset$ in $\langle U, N\rangle$ if and only if the following conditions are met:

(i) $S$ is closed under the rules in $N$. That is, if there is a rule $r \in N$ such that $r=\frac{\alpha_{1}, \ldots, \alpha_{n}: \beta_{1}, \ldots, \beta_{m}}{\varphi}, \alpha_{1}, \ldots, \alpha_{n} \in S$, and $\beta_{1}, \ldots, \beta_{m} \notin S$, then $\varphi$ belongs to $S$.

(ii) Whenever $\varphi \in S$ then there is a rule $r \in N$ such that $r=\frac{\alpha_{1}, \ldots, \alpha_{n}: \beta_{1}, \ldots, \beta_{m}}{\varphi}$, with $\alpha_{1}, \ldots, \alpha_{n} \in S, \beta_{1}, \ldots, \beta_{m} \notin S$

It is worth noting that deductively closed sets here play the role that Herbrand models of programs play in logic programming. Weak extensions here play a role similar to that of supported models of programs, that is models of Clark's completion, in logic programming. This analogy will become clearer when we discuss the semantic issues (see also Section 3 of part II). Here extensions of rule systems play a role analogous to that of stable models of logic programs in logic programming.

Following the analogy with logic programming, a set $S$ such that $T(S) \subseteq S$ is called a prefixpoint of $T$. Since $T$ is not, in general, a monotone operator, the Knaster-Tarski theorem cannot be applied. There is no guarantee that $T$ possesses a fixpoint. $U$ is, of course, a prefixpoint of $T$, so prefixpoints of $T$ always exist. Our remark that deductively closed sets are closed under intersections of descending 
families implies the following corollary by the Kuratowski - Zorn lemma:

Corollary 3.8 For every system $\langle U, N>$, and for every $S \subseteq U$ which is a prefixpoint of $T$, there is a minimal prefixpoint $S^{\prime}$ of $T, S^{\prime} \subseteq S$.

With each rule $r$ of form (2), we associate a monotonic rule of form (1)

$$
r^{\prime}=\frac{\alpha_{1}, \ldots, \alpha_{n}}{\varphi}
$$

obtained from $r$ by dropping all the restraints. Rule $r^{\prime}$ is called the projection of rule $r$. Let $N(S)$ be the collection of all $S$-applicable rules. That is, a rule $r$ belongs to $N(S)$ if all the premises of $r$ belong to $S$ and all restraints of $r$ are outside of $S$. We write $M(S)$ for the collection of all projections of all rules from $N(S)$. The projection $\left\langle U, N>\left.\right|_{S}\right.$ is the monotone system $\langle U, M(S)\rangle$. Thus $\left.\langle U, N\rangle\right|_{S}$ is obtained as follows: First, non-S-applicable rules are eliminated. Then, the restraints are dropped altogether. We have the following characterization theorem:

Theorem 3.9 A subset $S \subseteq U$ is an extension of $I$ in $\langle U, N\rangle$ if and only if $S$ is the deductive closure of I in $\left\langle U, N>\left.\right|_{S}\right.$.

Proof: If $S$ is an extension of $I$, then $S$ is closed under $S$-deductions. We can define a mapping from $S$-deductions from $I$ in $\langle U, N>$ to deductions from $I$ in the monotone system $<U, M(S)>$, by projecting the rules, that is, by dropping all restraints. By induction on the length of proofs we can show that none of the rules that were applied has been eliminated. Also, this map is surjective. This shows that if $S=C_{S}(I)$ (in $<U, N>$ ), then also $S=C(I)$ in $<U, M(S)>$. 
Conversely, every deduction of $\varphi$ in $\langle U, M(S)>$ can be lifted to an $S$-derivation of $\varphi$ in $\langle U, N\rangle$. An inductive argument shows that every $S$-derivation arises from lifting. This shows that $S=C(I)$ in $\langle U, M(S)>$ and implies that $S$ is an extension of $I$.

Actually, the projection procedure can be relaxed somewhat. Instead of eliminating all the rules that have a premise outside of $S$ or a restraint in $S$, it is enough to eliminate those that have restraints in $S$. Indeed, the rule $r=\frac{\alpha_{1}, \ldots, \alpha_{n}: \beta_{1}, \ldots, \beta_{m}}{\varphi}$ with an $\alpha_{i} \notin S$ is either inapplicable or, if it is applicable, then it witnesses the fact that the $C_{S}(I)$ is not $S$. Let $N^{+}(S)$ be the collection of rules in $N$ whose restraints are all outside of $S$, and let $\left\langle U, M^{+}(S)>\right.$ be the projection of $\left\langle U, N^{+}(S)\right\rangle$.

Proposition 3.10 $S \subseteq U$ is an extension of $I$ if and only if $S$ is the set of all consequences of I in the monotonic system $<U, M^{+}(S)>$.

The "relaxed" procedure for generating suitable collection of monotonic rules is closely connected to the Gelfond-Lifschitz procedure ([1988]) for stable models of logic programs. The "tight" procedure is a generalization of the Truszczynski procedure, [Marek and Truszczyński, 1989b].

Theorem 3.9 tells us how to test if a collection $S \subseteq U$ is an extension of $I$ in $<U, N>$. In case $U$ and $N$ are finite this leads to an algorithm.

(1) Compute $N(S)$.

(2) Project $N(S)$ by dropping restraints to get $M(S)$.

(3) Compute the deductive closure of $I$ in $\langle U, M(S)\rangle$, call this $T$. 
(4) Test whether $T=S$.

Finding all the extensions of a given $I$ is a complicated problem. A brute force algorithm is to generate all subsets of $U$, and test each of them for being an extension using the procedure above. A useful fact for improving this algorithm is:

Proposition 3.11 If $S$ is a extension of $I$, then $S$ consists entirely of elements of $I$ and conclusions of certain rules in $N$.

Thus an element of $U$ that is not a conclusion of any rule is never in an extension.

A simple construction allows us to consider only extensions of the empty set. In fact, if $\mathcal{S}$ is a nonmonotonic rule system, and $I \subseteq U$, then the system $\mathcal{S}(I)$ arises from

$\mathcal{S}$ and $I$ by adding to $N$ all the rules of the form $\frac{:}{\vartheta}$ for all $\vartheta \in I$. We then have:

Proposition $3.12 T$ is an extension of $I$ in $\mathcal{S}$ if and only if $T$ is an extension of $\emptyset$ in $\mathcal{S}(I)$.

Consequently, we shall consider below extensions of $\emptyset$. We say that $T$ is an extension of $\mathcal{S}$ if $T$ is an extension of $\emptyset$ in $\mathcal{S}$.

\section{Examples and Applications in Logic, Logic Pro- gramming, and Commonsense Reasoning}

\subsection{Classical Implicational Propositional Logic}

Here the set $U$ is the collection of all well-formed formulas of propositional logic, over some collection $A t$ of atoms with binary connective $\Rightarrow$ and constant $\perp$. The standard 
Lukasiewicz axiomatization is represented as a collection of rules of the form:

$$
\begin{gathered}
\frac{:}{\varphi \Rightarrow(\psi \Rightarrow \varphi)} \\
\frac{:}{(\varphi \Rightarrow(\psi \Rightarrow \vartheta)) \Rightarrow((\varphi \Rightarrow \psi) \Rightarrow((\varphi \Rightarrow \vartheta))} \\
\frac{:}{((\varphi \Rightarrow \perp) \Rightarrow(\psi \Rightarrow \perp)) \Rightarrow((\varphi \Rightarrow \perp) \Rightarrow \psi) \Rightarrow \varphi)} \\
\frac{\varphi, \varphi \Rightarrow \psi:}{\psi}
\end{gathered}
$$

The collection of derivable elements of $U$ is the set of tautologies of propositional logic. To represent the least fixed point of the operator associated with this system by the tableaux method requires the introduction of additional rules and the elimination of modus ponens (the fourth group) as a rule.

\subsection{Other Classical Systems}

Propositional logic may be represented in other ways as well, for instance in the language with the usual connectives $\neg, \wedge, \vee, \Rightarrow$. The collection $U$ then consists of all well-formed formulas of such a language. The exact set of rules depends on the actual axiomatization adopted. Intuitionistic logic may be similarly represented as a rule system using appropriate axioms. Likewise for modal logics.

\subsection{Ordinary Clausal Logic Programming}

Here $U$ is defined as follows: First, we have a fixed collection $A t$ of atoms. $U$ is the set of all pairs $\langle a, L>$ such that $a \in A t$, and $L$ is a finite subset of $A t(L=\emptyset$ is allowed). Thus, a clause $p \leftarrow q_{1}, \ldots, q_{r}$ is represented as a pair $\left\langle p,\left\{q_{1}, \ldots, q_{r}\right\}\right\rangle$. A 
logic program is a collection consisting of two lists of rules:

(1) (Specific rules) Rules of form $\frac{\dot{\varphi}}{\varphi}$ for $\varphi \in P$ where $P \subseteq U$.

(2) (Processing rules) Rules of form:

$$
\frac{<\alpha, \emptyset>,<\beta, L>}{<\beta, L \backslash\{\alpha\}>}
$$

for every $\alpha \in L$.

The pure PROLOG processing method, creating and processing goals, works here.

It is worth mentioning that, although on surface the same things happen here as in logic programming, in reality more is being computed, namely, all the proper Horn clauses derivable from the clauses in the "specific rules". It is, however, true that the atoms computed in this process constitute the least model of the program.

\subsection{Propositional Logic Programming, monotonic rules case}

Here the set $U$ consists of logical atoms. This system does not contain processing rules at all. The specific clauses are represented in a different fashion, namely as rules:

$$
\frac{\alpha_{1}, \ldots, \alpha_{n}:}{\varphi}
$$

with $n \geq 0$.

The resulting system is monotonic. The collection of objects computed here also consists only of atoms. 


\subsection{Default logic}

Again let $U$ be the collection of all formulas of propositional logic $\mathcal{L}$. Following Reiter

[1980] we introduce a default theory $\langle D, W\rangle$ as a pair where $D$ is a collection of default rules, that is, rules of form

$$
\frac{\alpha: \mathrm{M} \beta_{1}, \ldots, \mathrm{M} \beta_{m}}{\omega}
$$

(where $\alpha, \beta_{1}, \ldots, \beta_{m}$ are formulas) and $W$ a collection of formulas of the language $\mathcal{L}$.

Represent such a default theory as a rule system consisting of three lists:

(i) Elements of $\omega \in W$ are represented as rules:

$\frac{:}{\omega}$

(ii) Rules of form (4) are represented as

$$
\frac{\alpha: \neg \beta_{1}, \ldots, \neg \beta_{m}}{\omega}
$$

(That is, the restraints of the rule representing a default rule $r$ have an additional negation in front).

(iii) Processing rules of logic. That is, all the monotonic rules of the system of classical logic.

We then have the following proposition:

Proposition 4.1 A collection $S \subseteq U$ is an extension of a system consisting of rules of type (i), (ii), and (iii) if and only if $S$ is a default extension of $\langle D, W\rangle$.

Computing all extensions of a default theory can be achieved using the techniques developed in Section 4, part II. Notice that during the computation we need to test 
if the rule is $S$-applicable. This requires checking if $\alpha \in S, \neg \beta_{1}, \ldots, \neg \beta_{m} \notin S$. This can be carried out by the ordinary tableaux method, or any other complete theorem prover. These remarks are the source of the algorithm of [Marek and Nerode, 1990], in which a version specifically tailored to default logic is developed.

\subsection{Propositional logic programming, general case}

A general logic program is a list of general clauses, of the form:

$$
p \leftarrow q_{1}, \ldots, q_{n}, \neg r_{1}, \ldots, \neg r_{m}
$$

We refer to [Gelfond and Lifschitz, 1988] for the definition of a stable model of such

a program. That concept is a generalization of the perfect models as introduced in [Apt, Blair and Walker, 1987].

Let $U$ be the collection of atoms under consideration. Represent a general clause as a rule:

$$
\frac{q_{1}, \ldots, q_{n}: r_{1}, \ldots, r_{m}}{p}
$$

The translation $\operatorname{tr}(P)$ of a program $P$ is the set of translations of its individual clauses.

The following result was proved in [Bidoit and Froidevaux, 1988] and [Marek and Truszczyński, 1989b]:

Proposition 4.2 A subset $M \subseteq U$ is a stable model of $P$ if and only if $M$ is an extension of $\operatorname{tr}(P)$.

However weak extensions correspond to so-called supported models of $P$. (cf. Apt [Apt, 1988]) 
Proposition 4.3 A subset $M \subseteq U$ is a supported model of $P$ if and only if $M$ is a weak extension of $\operatorname{tr}(P)$.

\subsection{Logic programming with classical negation}

We now discuss the so-called "logic programming with classical negation" of [Gelfond and Lifschitz, 1989] as a chapter in the theory of nonmonotonic formal systems.

Recall the basic notions introduced in [Gelfond and Lifschitz, 1989]. The collection of objects appearing in heads or bodies of clauses is the set of all literals, that is, atoms or negated atoms. In particular, a negated atom may appear in the head of a clause. Consider first "general Horn" clauses in which literals may appear in arbitrary places. To each set $P$ of such clauses assign its answer set, the least collection $A$ of literals satisfying the following two conditions:

(1) If $a \leftarrow b_{1}, \ldots, b_{m}$ is in $P$ and $b_{1}, \ldots, b_{m} \in A$ then $a \in A$.

(2) If for some atom $p, p$ and $\neg p$ are both in $A$, then $A$ is the whole collection Lit of all literals.

Introduce a collection Str of structural processing rules over the set $U=$ Lit. These are all monotone rules of the form:

$$
\frac{p, \neg p:}{a}
$$

for all atoms $p$ and literals $a$.

Translate the clause: $a \leftarrow b_{1}, \ldots, b_{n}$ as rule:

$$
\frac{b_{1}, \ldots, b_{n}:}{a}
$$


and let $\operatorname{tr}(\mathrm{P})$ be the collection of translations of clauses in $P$ plus the structural rules Str. Then we have

Proposition $4.4 A$ subset $A \subseteq$ Lit is an answer set for $P$ if and only if $A$ is an extension of $\operatorname{tr}(P)$. Since $\operatorname{tr}(P)$ is a set of monotonic rules, such an answer set is the least fixpoint of the (monotonic) operator associated with the translation.

Gelfond and Lifschitz then introduce general rules. Since the negation used in literals is not the "negation-as-failure" of general logic programming, Gelfond and Lifschitz introduce another negation symbol "not" and a general logic clause with classical negation in the form:

$$
a \leftarrow b_{1}, \ldots, b_{n}, \operatorname{not}\left(c_{1}\right), \ldots, \operatorname{not}\left(c_{m}\right)
$$

Then the answer set for a set $P$ of clauses of this form is introduced by merging the operational procedure for the construction of stable models for a program (as introduced in [Gelfond and Lifschitz, 1988]) with the procedure above. They define the answer set for a program with classical negation as follows:

Let $M \subseteq$ Lit and $P$ be a general program. Define $P / M$ as a collection of clauses lacking not obtained as follows:

(1) If a clause $C$ contains a substring $\operatorname{not}(a)$ where $a \in M$, then eliminate $C$ altogether.

(2) In remaining clauses eliminate all substrings of the form $\operatorname{not}(a)$.

The resulting program $P / M$ lacks the symbol not, so the answer set is well defined. Let $M^{\prime}$ be the answer set for $P / M$. We call $M$ an answer set for $P$ precisely when 
$M^{\prime}=M$

Gelfond and Lifschitz give a computational procedure for finding such answer sets, and subsequently reduce computing them to computing default logic extensions. Here we give a general result showing that the construction of Gelfond and Lifschitz is faithfully represented within nonmonotonic rule systems; here is how. Define $U$ to be Lit, and translate the clause:

$$
a \leftarrow b_{1}, \ldots, b_{n}, \operatorname{not}\left(c_{1}\right), \ldots, \operatorname{not}\left(c_{m}\right)
$$

as the rule:

$$
\frac{b_{1}, \ldots, b_{n}: c_{1}, \ldots, c_{m}}{a}
$$

The translation of the program $P$ then consists of the translations of individual clauses $C$ of $P$, incremented by the structural rules Str. We get the following result:

Proposition 4.5 Let $P$ be a general logic program with classical negation and $N_{P}$ be the translation described above. Then a collection $M$ is an answer set for $P$ if and only if $M$ is an extension for the rule system $<U, N_{P}>$.

A number of facts proved in [Gelfond and Lifschitz, 1989] then become corollaries of the theory outlined here. Also, our results show that we get the same answer set if we tighten the operational definition of answer set for a general program, and change condition (1) to:

$\left(1^{\prime}\right)$ If $C$ contains in the body a literal $a$ which is not preceded by not and does not belong to $M$ or it contains a string not $(b)$ with $b$ belonging to $M$, then eliminate $C$ 
altogether.

((2) is left unaltered.)

\section{Representation of Combinatorial and Algebraic Problems as Structures associated with Rule Sys- tems}

The next set of examples show that the notions of extension, weak extension, and deductively closed set for nonmonotonic rule systems occur naturally throughout mathematics, particularly in combinatorics. This connection of nonmonotonic theories with everyday mathematics was not previously known.

\subsection{The Marriage Problem}

A society, $\mathcal{S}=<B, G, K>$ is a set $B$ of boys, a set $G$ of girls such that $B \cap G=\emptyset$, and a relation $K \subseteq B \times G$, the intended meaning of $<b, g\rangle \in K$ being " $b$ knows $g$ ". A marriage for a society $\mathcal{S}$ is a map $M: B \rightarrow G$. A marriage $M$ is proper if $M$ is one-to-one and for all $b \in B, M(b)=g$ implies $K(b, g)$. That is, in a proper marriage each boy marries a girl he knows. A marriage $M$ is symmetric if $M$ maps $B$ onto $G$. In a symmetric marriage, every girl is married.

For finite societies Philip Hall ([1935]) gave a necessary and sufficient condition for the existence of a proper marriage, namely:

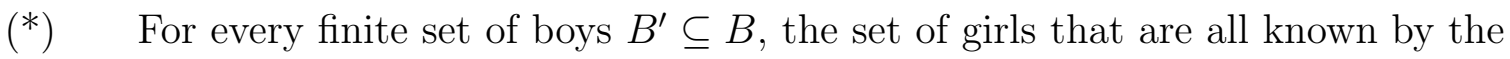
boys of $B^{\prime}$ has cardinality greater or equal than that of $B^{\prime}$. 
Marshall Hall ([1948]) showed that condition $\left(^{*}\right)$ is also a necessary and sufficient condition for the existence of marriages in an infinite society $\mathcal{S}$ as long as each boy knows only finitely many girls. Philip Hall's theorem is a special case of a more general theorem for finding transversals (see [Mirsky, 1971]).

We claim that if $\mathcal{S}=<B, G, K>$ is a society satisfying (*) in which each boy knows only finitely many girls, then there is a nonmonotonic rule system $\mathcal{Z}=<$ $U(\mathcal{S}), N(\mathcal{S})>$ such that the collection of extensions of $\mathcal{Z}$ correspond exactly to the set of proper marriages of $\mathcal{S}$. To this end let us consider a collection of strings $U(\mathcal{S})=\{M b g: b \in B, g \in G$, and $K(b, g)$ holds $\}$, where $M$ is a new symbol. Then for each boy $b \in B$ if $\left\{g_{1}, \ldots, g_{n}\right\}$ is the set of girls $b$ knows, we add the following set of $n$ rules to $N(\mathcal{S})$.

$$
\frac{: M b g_{1}, \ldots, \widehat{M b g}_{k}, \ldots, M b g_{n}}{M b g_{k}}
$$

where we adopt the convention that for any $n$-element sequence $s_{1}, \ldots, s_{n}$, let $s_{1}, \ldots, \hat{s_{k}}$, $\ldots, s_{n}$ be the $n-1$-element sequence that results from $s_{1}, \ldots, s_{n}$ by removing $s_{k}$. For any girl $g$ and any two boys $b_{1} \neq b_{2}$, each of whom knows $g$, add the following rules to $N(\mathcal{S})$ :

$$
\frac{M b_{1} g, M b_{2} g:}{\varphi}
$$

for every $\varphi \in U(\mathcal{S})$. Let $N(\mathcal{S})$ consist of all the rules of the form $(5)$ or $(6)$.

Theorem 5.1 Let $\mathcal{S}=<B, G, K>$ be a society satisfying (*), for which each boy knows only finitely many girls. Then $E$ is an extension for $\mathcal{Z}=<U(\mathcal{S}), N(\mathcal{S})>$ if and only if $M_{E}=\{\langle b, g\rangle: M b g \in E\}$ is a proper marriage for $\mathcal{S}$. 
Proof. $(\Rightarrow)$ First, observe that if $b \in B$ knows only one girl $g$, then the rule $\frac{:}{M b g}$ belongs to $N=N(\mathcal{S})$, so that $M b g \in E$ for any extension $E$. Let $B_{1} \subseteq B$ be the set of boys in $B$ who know only one girl. Since $\mathcal{S}$ satisfies condition $\left(^{*}\right)$, we know that there is a proper marriage for $\mathcal{S}$, so that there can be no girl $g$ such that there exist boys $b_{1}$ and $b_{2}$ in $B_{1}$ for whom $M b_{1} g$ and $M b_{2} g$ are in $U=U(\mathcal{S})$. Hence if we can apply rules of the form (6), at least one of $M b_{1} g$ and $M b_{2} g$ is not derived from an axiom. This means that, if $B_{1}=B$, then the only extension $E$ of $\mathcal{Z}$ is $U$ and $M_{U}$ is the unique proper marriage of $\mathcal{S}$. So assume $B_{1} \neq B$. Then we claim that $U$ is not an extension of $\mathcal{Z}$. Note that if $b \in B \backslash B_{1}$ then application of all rules of the form (5) for $b$ is blocked by $U$. It then follows easily from our observation about the applicability of rules of the form (6) that we can never derive both the premises of rules of the form (6) from $\emptyset$. Thus $C_{U}(\emptyset)=\left\{M b g \in U: b \in B_{1}\right\} \neq U$.

Since $U$ is not an extension, for any extension $E$ there cannot be $b_{1}, b_{2} \in B$ and $g \in G$ such that $M b_{1} g$ and $M b_{2} g$ are in $E$, since otherwise the rules of the form (6) would show $E \subseteq C_{E}(\emptyset) \Rightarrow C_{E}(\emptyset)=U$. This automatically means that the map $M_{E}$ is one-to-one. Since we can only use rules of the form (5) to derive elements of $C_{E}(\emptyset)$ for an extension $E$, it can not be the case that if $b \in B-B_{1}$, then there exist $g_{i} \neq g_{j}$ in $G$ such that $M b g_{i}$ and $M b g_{j}$ belong to $E$, for then either $M b g_{i}$ or $M b g_{j}$ would block the application of any rule of form (5) for $b$. But then neither $M b g_{i}$ nor $M b g_{j}$ can be in $C_{E}(\emptyset)$, so $C_{E}(\emptyset) \neq E$. Also, if $b \in B$ we have to have at least one $M b g_{i}$ in $E$, otherwise all the rules of form (5) for $b$ apply for $E$ and hence $M b g_{i} \in C_{E}(\emptyset) \backslash E$ for all $i$. Thus for each $b \in B$, there is exactly one $g$ such that $M b g \in E$. Thus $M_{E}$ is 
a one-to-one function from $B$ to $G$. Finally notice that $M_{E}$ is proper, because by our definition, $M b g \in U$ implies that $K(b, g)$ holds. Thus if $E$ is an extension, $M$ defines a proper marriage.

$(\Leftarrow)$ Suppose $M$ is a proper marriage for $\mathcal{S}$ and $E=\{M b g: M(b)=g\}$. Then clearly, $M_{E}=M$. It is easy to see that rules of the form (6) allow us to derive $M b g$ precisely when $M(b)=g$. Moreover we can never apply a rule of the form (6) without at some point using rules of the form (5) to derive both premises of some rule of the form (6). Since $M$ is a proper marriage, we can never derive $M b_{1} g$ and $M b_{2} g$ from rules of the form $(5)$ if $b_{1} \neq b_{2}$. Thus $C_{E}(\emptyset)=E$ and $E$ is an extension.

We should observe that for our $\mathcal{Z}=<\mathcal{U}(\mathcal{S}), \mathcal{N}(\mathcal{S})>$, the only weak extension which is not an extension is $U(\mathcal{S})$. That is, if $E \subseteq U$ and any rule of the form (6) is applicable for $E$, then

$$
R=\left\{\varphi: \exists_{r \in N}\left(r=\frac{\alpha_{1}, \ldots, \alpha_{n}: \beta_{1}, \ldots, \beta_{m}}{\varphi} \wedge \alpha_{1}, \ldots, \alpha_{n} \in E \wedge \beta_{1}, \ldots, \beta_{m} \notin E\right)\right\}=U
$$

So that $C_{E}(R)=U$. If no rule of the form (6) is $E$-applicable, and $E$ is a weak extension, then the same argument used in the proof of Theorem 5.1 shows that $M_{E}$ must be a proper marriage. Finally, observe that if $D$ is deductively closed and $D \neq U$, then none of the rules of (6) are $D$-applicable. Moreover, it will be still the case that for each boy $b \in B$, the rules of the form (5) will force at least one $M b g_{i} \in D$. However it is possible to have $M b g_{i}$ and $M b g_{j}$ in $D$ with $g_{i} \neq g_{j}$, in which case none of the rules of the form (6) for $b$ force elements into $D$. Thus deductively closed sets $D \neq U$ correspond to marriages in which each boy marries at least one girl he knows and may marry several girls he knows, but no girl is married to more than 
one boy. Consequently, minimal deductively closed sets correspond again to proper marriages.

By expanding our set of rules $N(\mathcal{S})$, we can ensure that extensions correspond to proper symmetric marriages. That is, suppose that $\mathcal{S}=<B, G, K>$ is a society in which every boy knows only finitely many girls, and every girl knows only finitely many boys, and there is a symmetric marriage for $\mathcal{S}$. Let $U$ be defined as before. In addition to all rules of form (5) and (6), add a set of rules for each $g \in G$.

If $g \in G$ and $\left\{b_{1}, \ldots, b_{n}\right\}$ is the set of boys that $g$ knows, then add the following set of rules:

$$
\frac{: M b_{1} g, \ldots, \widehat{M b_{k}} g, \ldots, M b_{n} g}{M b_{k} g}
$$

Let $N_{\text {Sym }}(\mathcal{S})$ be the collection of rules of form $(5),(6)$, and $(7)$ and let $U_{\text {Sym }}(\mathcal{S})=U$. By a proof which is similar to that of Theorem 5.1, we can prove the following:

Theorem 5.2 Let $\mathcal{S}=<B, G, K>$ be a society such that each boy knows only finitely many girls and each girl knows only finitely many boys, and there is a proper symmetric marriage for $\mathcal{S}$. Then $E$ is an extension for $\mathcal{Z}_{\text {Sym }}=<U_{\text {Sym }}(\mathcal{S}), N_{\text {Sym }}(\mathcal{S})>$ if and only if $M_{E}=\{\langle b, g\rangle: M b g \in E\}$ is a proper symmetric marriage for $\mathcal{S}$.

\subsection{Proper $k$-colorings of graphs}

Let $\mathcal{G}=<V, E>$ be a graph, where $V$ is the set of vertices of $\mathcal{G}$, and $E$ is the set of edges of $\mathcal{G}$. For $x \in V, N b(x)=\{y \in V:<x, y>\in E\}$ denotes the set of neighbors of $x$ in $\mathcal{G}$. We say that $\mathcal{G}$ is locally finite if $N b(x)$ is finite for all $x \in X$. A map $C: V \rightarrow\{1, \ldots, k\}$ is called a proper $k$-coloring if whenever $<x, y>\in E$, then 
$C(x) \neq C(y)$.

We claim that for any locally finite graph $\mathcal{G}=\langle V, E\rangle$, there is a nonmonotonic rule system $\mathcal{S}=<U(\mathcal{G}), N(\mathcal{G})>$ such that the extensions of $\mathcal{S}$ correspond to the proper $k$-colorings of $\mathcal{G}$. Let $U(\mathcal{G})=\{C x i: x \in V \wedge i \in\{1, \ldots, k\}\}$. Then, for each $x \in V$, we add the following set of rules to $N(\mathcal{G})$.

$$
\frac{: C x 1, \ldots, \widehat{C x} j, \ldots, C x k}{C x j}
$$

for $j \in\{1, \ldots, k\}$. Also, for each edge $<x, y>\in E$ and $i \in\{1, \ldots, k\}$ we add the following set of rules to $N(\mathcal{G})$.

$$
\frac{C x i, C y i:}{\varphi}
$$

for all $\varphi \in U(\mathcal{G})$.

By a proof which is very similar to that of Theorem 5.1, we can prove the following

Theorem 5.3 Let $\mathcal{G}=\langle V, E>$ be a locally finite graph. Then $E$ is an extension for $\left\langle U(\mathcal{G}), N(\mathcal{G})>\right.$ if and only if $C_{E}=\{\langle x, i\rangle$ :Cxi $\in E\}$ is a proper $k$-coloring of $\mathcal{G}$.

Again we can show that the only weak extension of $<U(\mathcal{G}), N(\mathcal{G})>$ which is not an extension is $U(\mathcal{G})$ itself. Also we can show that the deductively closed sets $D \subseteq U(\mathcal{G})$ correspond to the colorings for which each vertex is assigned at least one, but possibly many, colors and if $\langle x, y\rangle \in E$, then it cannot be the case that there is a color $i$ that is assigned to both $x$ and $y$. 


\subsection{Chain Covers of Partially Ordered Sets}

Let $\mathcal{P}=<D, \leq_{D}>$ be a partially ordered set, that is $\leq_{D}$ is a binary relation on $D$ such that

(i) $\forall_{x \in D} x \leq{ }_{D} x$,

(ii) $\forall_{x, y, z \in D}\left(x \leq_{D} y \wedge y \leq_{D} z \Rightarrow x \leq_{D} z\right)$,

(iii) $\forall_{x, y \in D}\left(x \leq_{D} y \wedge y \leq_{D} x \Rightarrow x=y\right)$.

For $x, y \in D$ we say that $x$ is incomparable to $y$, written $x \mid y$, if neither $x \leq_{D} y$ nor $y \leq_{D} x$. We say that a set $A \subseteq D$ is an antichain if whenever $x \neq y$ and $x, y \in A$ then $x \mid y$. We define the width of $\mathcal{P}$ to be $w$ if the maximum size of an antichain is $w$. We say that a set $C \subseteq D$ is a chain if whenever $x, y \in C$, then either $x \leq_{D} y$ or $y \leq_{D} x$.

We say that a set of chains $\left\{C_{1}, \ldots, C_{n}\right\}$ is a chain cover of $\mathcal{P}$ or that $\mathcal{P}$ is covered by $C_{1}, \ldots, C_{n}$ if $D=\bigcup_{i=1}^{n} C_{i}$. We say that a chain cover of $\mathcal{P}$ is disjoint if the chains are pairwise disjoint. Clearly any chain cover for $\mathcal{P}$ can be refined to a disjoint chain cover for $\mathcal{P}$. Dilworth [1950] proved the following fact for finite partially ordered sets:

Proposition 5.4 If $\mathcal{P}=<D, \leq_{D}>$ is a partially ordered set of width $w$, then there are $w$ chains $C_{1}, \ldots, C_{w}$ in $\mathcal{P}$ which cover $\mathcal{P}$.

Dilworth's Theorem can be extended for partially ordered sets of arbitrary cardinality by applying the Compactness Theorem for propositional logic. Then for any partially ordered set $\mathcal{P}=\left\langle D, \leq_{D}>\right.$ of width $w$, we can define a rule system $\langle U(\mathcal{P}), N(\mathcal{P})\rangle$ such that the extensions of $<U(\mathcal{P}), N(\mathcal{P})>$ correspond to disjoint chain covers 
of $\mathcal{P}$ by $w$ chains $C_{1}, \ldots, C_{w}$. Note that for disjoint chain covers $C_{1}, \ldots, C_{w}$ of $\mathcal{P}$, there is a natural map $\mathcal{C} \rightarrow\{1, \ldots, w\}$ defined by $\mathcal{C}(x)=i$ if and only if $x \in C_{i}$. Thus, defining a disjoint chain cover is just like coloring the elements of $D$ with one of $w$ colors, while ensuring that $x$ and $y$ are colored differently if $x \mid y$. Let $U(\mathcal{P})=\{C x i: x \in D \wedge i \in\{1, \ldots, w\}\}$. For each $x \in D$, we add the following set of rules to $N(\mathcal{P})$.

$$
\frac{: C x 1, \ldots, \widehat{C x} j, \ldots, C x w}{C x j}
$$

for $j \in\{1, \ldots, w\}$. For each $x, y \in D$ such that $x \mid y$ and each $i \in\{1, \ldots, w\}$ we add the following rules to $N(\mathcal{P})$.

$$
\frac{C x i, C y i:}{\varphi}
$$

for all $\varphi \in U(\mathcal{P})$.

By a proof which is very similar to the proof of Theorem 5.1, we can prove the following

Theorem 5.5 Let $\mathcal{P}=<D, \leq_{D}>$ be a partially ordered set of width $w$. Then $E$ is an extension for $\langle U(\mathcal{P}), N(\mathcal{P})\rangle$ if and only if $\left\langle C_{1}, \ldots C_{w}\right\rangle$ is a disjoint chain cover of $\mathcal{P}$ (where for $\left.i \in\{1, \ldots, w\}, C_{i}=\{x \in D: C x i \in E\}\right)$.

Again we can show that the only weak extension of $\langle U(\mathcal{P}), N(\mathcal{P})\rangle$ which is not an extension is $U(\mathcal{P})$. Also we can show that the deductively closed sets $D \neq U(\mathcal{P})$ correspond to assignments in which each $x \in D$ is assigned to at least one, but possibly many, chains, and if $x \mid y$, then $x$ and $y$ are not assigned to the same chain. Thus deductively closed sets $D \neq U(\mathcal{P})$ correspond to arbitrary chain covers of $\mathcal{P}$. 
The above examples are just a few of many examples where extensions correspond to "solutions" of certain natural combinatorial problems. Next, we turn to problems in algebra where extensions naturally occur.

\subsection{Maximal Ideals in a Boolean Algebra}

Let $\mathcal{B}=<B, \wedge, \vee, \neg, 0_{\mathcal{B}}, 1_{\mathcal{B}}>$ be a Boolean Algebra, where $\wedge, \vee$, and $\neg$ are the operations of meet, join, and complement for $\mathcal{B}$, and $0_{\mathcal{B}}$ and $1_{\mathcal{B}}$ denote the zero and unit of $\mathcal{B}$ respectively. We let $\leq_{\mathcal{B}}$ denote the order induced by $\mathcal{B}$. That is, $x \leq_{\mathcal{B}} y$ if and only if $x \wedge y=x$.

$I \subseteq \mathcal{B}$ is an ideal of $\mathcal{B}$ if

(i) $0_{\mathcal{B}} \in I$,

(ii) $\forall_{x, y \in B} x \leq_{\mathcal{B}} y \wedge y \in I \Rightarrow x \in I$, and

(iii) $\forall_{x, y \in B} x \in I \wedge y \in I \Rightarrow x \vee y \in I$.

An ideal $I$ is maximal if for any $x \in B$ exactly one of $x$ and $\neg x$ is in $I$.

Given any Boolean Algebra $\mathcal{B}=<B, \wedge, \vee, \neg, 0_{\mathcal{B}}, 1_{\mathcal{B}}>$ we can give a nonmonotonic rule system $<U(\mathcal{B}), N(\mathcal{B})>$ such that the extensions of $<U(\mathcal{B}),<N(\mathcal{B})>$ correspond exactly to maximal ideals of $\mathcal{B}$. Let $U(\mathcal{B})=B$. Then $N(\mathcal{B})$ consists of the following five classes of rules:

$$
\begin{gathered}
\frac{:}{0_{\mathcal{B}}} \\
\frac{: x}{\neg x}
\end{gathered}
$$


for all $x \in B$.

$$
\frac{x, \neg x:}{y}
$$

for all $x, y \in B$.

$$
\frac{x:}{y}
$$

for all $x, y \in B$ whenever $y \leq_{\mathcal{B}} x$.

$$
\frac{x, y:}{x \vee y}
$$

for all $x, y \in B$.

Theorem 5.6 Let $\mathcal{B}=<B, \wedge, \vee, \neg, 0_{\mathcal{B}}, 1_{\mathcal{B}}>$ be a Boolean Algebra. Then $E$ is an extension of $<U(\mathcal{B}), N(\mathcal{B})>$ if and only if $E$ is a maximal ideal of $\mathcal{B}$.

Proof: $(\Rightarrow)$ Suppose that $E$ is an extension of $<U(\mathcal{B}), N(\mathcal{B})>$. Then since $C_{E}(\emptyset)=$ $E$, it is easy to see that the rules of form (12), (15) and (16) ensure that $E$ is an ideal of $\mathcal{B}$. Rules of form (13) ensure that at least one of $x$ and $\neg x$ is in $E$ for all $x \in B$. If for some $x \in B$ both $x$ and $\neg x$ are in $E$, then rules of the form (14) ensure $E=U(\mathcal{B})=B$. Thus if $E \neq B$, then exactly one of $x$ and $\neg x$ is in $E$ for each $x \in B$. Hence $E$ is a maximal ideal. Thus we need only to show that $U(\mathcal{B})=B$ is not an extension. Note that all rules of the form (13) are blocked for $B$. Thus the only element we can derive to be in $C_{B}(\emptyset)$ with no premises is $0_{\mathcal{B}}$. But starting with $0_{\mathcal{B}}$, we can not apply rules (15) but only apply rules (15) where $x=0_{\mathcal{B}}$, or rules (16) where $x=y=0_{\mathcal{B}}$, and such rules give $0_{\mathcal{B}}$ as conclusion. Then, by an easy induction on the length of proofs, we can show that $C_{B}(\emptyset)=\left\{0_{\mathcal{B}}\right\}$ so that $B$ is not an extension. $(\Leftarrow)$ Note that if $E$ is a maximal ideal, then rules of the form (13) ensure $E \subseteq C_{E}(\emptyset)$. 
Successive applications of rules (12), (15), and (16) will produce no new elements. Then we can prove by induction on the length of proofs that we can never apply rules of the form (14), hence $C_{E}(\emptyset) \subseteq E$.

Recall that $F$ is a (maximal) filter of $\mathcal{B}$ if and only if $\{x \in B: \neg x \in F\}$ is a (maximal) ideal in $\mathcal{B}$. Clearly we can set up a nonmonotonic rule system < $U_{f i l}(\mathcal{B}), N_{f i l}(\mathcal{B})>$ such that extensions of $<U_{f i l}(\mathcal{B}), N_{f i l}(\mathcal{B})>$ correspond to maximal filters of $\mathcal{B}$. Now, it is well known that a propositional logic modulo logical equivalence is a Boolean Algebra $\mathcal{B}$ and that the maximal filters correspond to the complete theories. Thus we can also set up a nonmonotonic rule system $\langle U, N\rangle$ whose extensions correspond to complete theories of a propositional logic.

\subsection{Complementary Subspaces of Vector Spaces}

Let $V_{\infty}$ be a countably infinite dimensional vector space over a finite field $F$, and let $B=\left\{b_{0}, b_{1}, \ldots\right\}$ be a basis for $V_{\infty}$. If $S \subseteq V_{\infty}$, we let $(S)^{\star}$ denote the space generated by $S$. Let $V_{n}=\left(\left\{b_{0}, \ldots, b_{n}\right\}\right)^{\star}$ for $n \geq 1$. Given two subspaces $A$ and $B$ of $V_{\infty}$, we write $A+B$ for $(A \cup B)^{\star}$ and $A \oplus B$ for $A+B$ if $A \cap B=\{0\}$, where 0 is zero vector of $V_{\infty}$.

Now, suppose that $W$ is a subspace of $V_{\infty}$. We claim that we can define a nonmonotonic rule system $<U, N>=<U_{W}\left(V_{\infty}\right), N_{W}\left(V_{\infty}\right)>$ so that extensions of $\langle U, N>$ correspond to the complementary subspaces for $W$ that arise from the most natural construction of such spaces. That is, if one were going to construct a subspace $A$ such that $A \oplus W=V_{\infty}$, a natural way to proceed would be to construct a sequence 
of subspaces $A_{0} \subseteq A_{1} \subseteq \ldots$ in stages as follows:

Stage 0 Let $A_{0}=\{0\}$.

Stage $\mathbf{s}+\mathbf{1}$ Having defined a subspace $A_{s} \subseteq V_{s}$ such that $A_{s} \oplus W_{s}=V_{s}$ where $W_{n}=W \cap V_{n}$ for $n \geq 1$, we proceed according to one of two cases.

Case $1 W_{s}$ is properly included in $W_{s+1}$.

In this case, it is easy to show that $A_{s} \oplus W_{s+1}=V_{s+1}$, so we let $A_{s+1}=A_{s}$.

Case $2 W_{s}=W_{s+1}$.

In this case it is easy to show that if we choose $x_{s+1} \in V_{s+1} \backslash V_{s}$ and $A_{s+1}=$ $\left(A_{s} \cup\left\{x_{s+1}\right\}^{\star}\right)$, then $A_{s+1} \subseteq V_{s+1}$ and $A_{s+1} \oplus W_{s+1}=V_{s+1}$.

Then $A=\bigcup_{s} A_{s}$ is the desired complementary subspace of $W$. Note that we can get many different such complementary subspaces depending on the choice of $x_{s+1}$ at each stage in which Case 2 occurs.

We define our nonmonotonic rule system $<U, N>=<U_{W}\left(V_{\infty}\right), N_{W}\left(V_{\infty}\right)>$ as follows. We let $U=V_{\infty}$. Then we let $N$ consist of the following five classes of rules:

$$
\begin{gathered}
\frac{:}{0}, \\
\frac{x_{1}, \ldots, x_{k}:}{\sum_{i=1}^{k} \lambda_{i} x_{i}}
\end{gathered}
$$

for all $x_{1}, \ldots, x_{k} \in V_{\infty}$, and $\lambda_{1}, \ldots, \lambda_{k} \in F$,

$$
\frac{x:}{v}
$$

for all $x \in W \backslash\{0\}$ and $v \in V_{\infty}$,

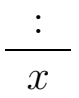


where $x \in V_{s_{0}}$ and $s_{0}$ is the largest $s$ such that $W_{s}=\{0\}$ and $W_{s+1} \neq\{0\}$, and

$$
\frac{a_{1}, \ldots, a_{n}: b_{1}, \ldots, b_{m}}{x}
$$

where $A=\left\{a_{1}, \ldots, a_{n}\right\}$ is a subspace and for some $s>s_{0}, A \oplus W_{s}=V_{s} ;\{0\} \neq$ $W_{s}=W_{s+1}, x \in V_{s+1} \backslash V_{s}$, and $\left\{b_{1}, \ldots, b_{m}\right\}=V_{s+1}-(A \cup\{x\})^{\star}$.

Theorem 5.7 Let $V_{\infty}$ be a countably infinite dimensional vector space over a finite field $F$, let $B=\left\{b_{1}, b_{2}, \ldots\right\}$ be a basis for $V_{\infty}$ and let $W$ be a subspace of $V_{\infty}$. Then $E$ is an extension of $\left\langle U, N>=<U_{W}\left(V_{\infty}\right), N_{W}\left(V_{\infty}\right)>\right.$ if and only if $E$ is a subspace such that $E \oplus W=V_{\infty}$ and for all $s \geq 1, E_{s} \oplus W_{s}=V_{s}$ (where $V_{s}=\left\{b_{1}, \ldots, b_{s}\right\}^{\star}$, $E_{s}=E \cap V_{s}$, and $\left.W_{s}=W \cap V_{s}\right)$.

Proof: $(\Rightarrow)$ Suppose that $E=C_{E}(\emptyset)$. First observe that the rules of the form $(17)$, (18), and (20) ensure that $E$ is a subspace of $V_{\infty}$ containing $V_{s_{0}}$. Next, observe that the rules (19) ensure that if $E \cap W \neq\{0\}$, then $E=V_{\infty}$. We claim that $E=V_{\infty}$ is impossible. For if $E=V_{\infty}$, then all the rules of the form (21) are blocked by $E$. The remaining rules are monotonic. So it is easy to prove by induction on the length of deductions that the only $x \in V_{\infty}$ which can be deduced are the $x \in V_{s_{0}}$. But then $C_{V_{\infty}}(\emptyset)=V_{s_{0}} \neq V_{\infty}$ so that $V_{\infty}$ is not an extension. Thus $E \neq V_{\infty}$, so by the rules (19) we conclude that $E \cap W=\{0\}$. Finally consider the rules of the form (21). We use these rules to prove by induction that for all $s \geq s_{0}, E_{s} \oplus W_{s}=V_{s}$, where $E_{s}=E \cap V_{s}$. First, we know $E_{s_{0}}=V_{s_{0}}$ and $W_{s_{0}}=\{0\}$ so that $E_{s_{0}} \oplus W_{s_{0}}=V_{s_{0}}$. Assume that $s \geq s_{0}$ and $E_{s} \oplus W_{s}=V_{s}$. Then if $W_{s} \subset W_{s+1}$, we know that $E_{s} \oplus W_{s+1}=V_{s+1}$. Since $E \cap W=\{0\}$, we must have $E_{s+1}=E \cap W_{s+1}=E_{s}$. If $W_{s}=W_{s+1}$, then we can 
apply rules of the form (21) to conclude that for some $x \in V_{s+1} \backslash V_{s}, x \in E$. But then $E_{s+1} \supset E_{s}$ and so $E_{s+1}+W_{s+1}=V_{s+1}$. But again since we know that $E \cap W=\{0\}$, we must conclude that $E_{s+1} \cap W_{s+1}=\{0\}$ so that $E_{s+1} \oplus W_{s+1}=V_{s+1}$.

$(\Leftarrow)$ Suppose that $E$ is a subspace of $V_{\infty}$ such that $E \oplus W=V_{\infty}$ and for all $s$, $E_{s} \oplus W_{s}=V_{s}$. It is easy to see that the rules (20) and (21) allow us to prove by induction on $s$ that $E_{s} \subseteq C_{E}(\emptyset)$ for all $s$. Moreover it is not difficult to show by induction on the length of deductions that if we can deduce that some $x \notin E$ is in $C_{E}(\emptyset)$, then, at some point in the deduction we must use a rule of the form (21) to derive that some $y \in C_{E}(\emptyset)$, where $y \notin E$. But all the rules of the form $(21)$ which have a $y$ as a conclusion (where $y \notin E$ ) are blocked. Thus, we conclude that $C_{E}(\emptyset) \subseteq E$. Hence $C_{E}(\emptyset)=E$.

We note that the example of subspaces forms a prototype for how nonmonotonic rule systems can be used to mirror stage constructions of substructures in a variety of algebraic situations.

\subsection{Prime ideals in a commutative ring $\mathcal{R}$, the radical and skeptical reasoning}

Let $\mathcal{R}=<R,+, \cdot, 0,1>$ be a commutative ring with unit. An ideal $I$ is an additive subgroup of $\mathcal{R}$ such that if $x \in I$ and $y \in R$ then $x y \in I$. I is said to be a prime ideal if $I \neq R$ and for all $x, y \in R$, if $x y \in I$, then either $x \in I$ or $y \in I$. The McCoy radical of $\mathcal{R}$ is the intersection of all prime ideals of $\mathcal{R}$. We shall construct a nonmonotonic rule system $<U(\mathcal{R}), N(\mathcal{R})>$ such that the set of extensions of $\langle U(\mathcal{R}), N(\mathcal{R})>$ is exactly the set of prime ideals of $\mathcal{R}$. Since prime ideals can be included one in 
another, while the extensions are always incomparable, we use a technique which allows us to represent both the ideal and its complement as an extension of a suitably chosen system.

Let $U(\mathcal{R})=\{x, \bar{x}: x \in R\} . \bar{x}$ is a new logical synbol that will be interpreted as indicating that $x$ does not belong to $I . N(\mathcal{R})$ consists of the following eight classes of rules:

$$
\frac{1:}{0} \quad \frac{1:}{z}
$$

for all $z \in U(\mathcal{R})$.

$$
\frac{y:}{-y}
$$

for all $y \in R$ (here $-y$ is the additive inverse of $y$ )

$$
\frac{x, y:}{x+y}
$$

for all $x, y \in R$.

$$
\frac{x:}{x y}
$$

for all $x, y \in R$.

$$
\frac{x y, \bar{x}:}{y} \quad \frac{x y, \bar{y}:}{x}
$$

for all $x, y \in R$.

$$
\frac{x y, \bar{x}, \bar{y}:}{z}
$$

for all $x, y \in R$ and $z \in U(\mathcal{R})$.

$$
\frac{: x}{\bar{x}} \quad \frac{: \bar{x}}{x}
$$

for all $x \in R$.

$$
\frac{x, \bar{x}:}{z}
$$


for all $x \in R$ and $z \in U(\mathcal{R})$.

Theorem 5.8 Let $\mathcal{R}=<R,+, \cdot, 0,1>$ be a commutative ring with a unit. Then $E$ is an extension of $\langle U(\mathcal{R}), N(\mathcal{R})>$ if and only if there is a prime ideal $I$ of $\mathcal{R}$ such that $E=\{x: x \in I\} \cup\{\bar{x}: x \in R \backslash I\}$.

Proof: $(\Rightarrow)$ Suppose that $E$ is an extension of $\langle U(\mathcal{R}), N(\mathcal{R})>$. First we show that $U(\mathcal{R})=U$ is not an extension of $\langle U(\mathcal{R}), N(\mathcal{R})>$. That is, it is easy to see that the only axiom is 0 and that derivation of any other element can be blocked. Then, by induction on the length of derivations we can prove that the only element we can generate starting with 0 by applying rules in $N(\mathcal{R})$ which are not blocked by $U$ is 0 itself. Thus $C_{U}(\emptyset)=\{0\} \neq U$.

Thus $E \neq U$. But then we see that rules of form (28) and (29) will force exactly one of $x$ or $\bar{x}$ to be in $E$ for each $x \in R$. Then let $I_{E}=\{x: x \in R \wedge x \in E\}$. Clearly rules of the form $(22)-(27)$ force that $I_{E}$ is a prime ideal. Thus $E=\{x: x \in$ $\left.I_{E}\right\} \cup\left\{\bar{x}: x \in R \backslash I_{E}\right\}$.

$(\Leftarrow)$ Now suppose that $I$ is a prime ideal of $\mathcal{R}$ and $E=\{x: x \in I\} \cup\{\bar{x}: x \in R \backslash I\}$. The rules of the form (28) ensure that $E \subseteq C_{E}(\emptyset)$. Then, a straightforward induction on the length of derivations shows that $C_{E}(\emptyset) \subseteq E$. Thus $E$ is an extension.

Finally, following the intuitions from nonmonotonic logics, if we compute the collection of nonmonotonic consequences of $<U(\mathcal{R}), N(\mathcal{R})>$ that is, the intersection of all extensions of $\langle U(\mathcal{R}), N(\mathcal{R})>$, then in the above case it corresponds to the radical of $\mathcal{R}$. Putting it more explicitely we get the collection $\{x: x \in J\} \cup\{\bar{x}: x \in W\}$ 
where $J$ is the radical of $\mathcal{R}$ and $W$ is the set of units of $\mathcal{R}$.

\section{Conclusions and the Contents of Part II}

This paper introduced the concept of a nonmonotonic rule system. We demonstrated that many existing forms of nonmonotonic reasoning due to Reiter (Default Logic), Doyle (Truth Maintenance), Gelfond and Lifschitz (Stable Semantics for Logic Programs), Clark (Negation as Failure), are naturally represented as nonmonotonic rule systems. We demonstrated that when these logics are interpreted as nonmonotonic rule systems, we get uniform treatments for concepts, theorems and algorithms. We also gave purely mathematical examples of nonmonotonic rule systems which show that nonmonotonic rule systems occur widely in mathematical practice.

In Part II we will investigate the recursive complexity of the class of all extensions (or weak extensions or deductively closed sets) of a nonmonotonic rule system. In Section 5 we proved that the set of all marriages for a "marriage problem" is represented exactly as the set of extensions in suitably chosen nonmonotonic rule systems. In Part II we prove a converse for "highly recursive" societies. Similar result is obtained for graph colorings. A more general result, for recursive rule systems, and for general rule systems will be published in Part III of this paper. The connection with "marriage problem" provides a wealth of recursive-theoretic results on extensions of highly recursive nonmonotonic rule systems and therefore many new results for the known nonmonotonic logics mentioned above.

In Part II we give an "infinitary" semantics for nonmonotonic systems in the logic 
$\mathcal{L}_{\omega_{1}, \omega}$ based on infinitary disjunction, conjunction and negation. In the case when the domain $U$ of the nonmonotonic system is $\omega$, using the arithmetical hierarchy of functions as in Rogers [1967], we find that the class of all extensions, weak extensions, closed sets and minimal closed sets. These classes are, respectively, $\Pi_{2}^{0, N}$ (effectively $\left.G_{\delta}\right), \Pi_{2}^{0, N}, \Pi_{1}^{0, N}$ (effectively closed), and $\Pi_{2}^{0, N}$. We get various results on Reiter's default theories, and Gelfond and Lifschitz stable models of general logic programs.

Finally, we discuss various algorithms of computing extensions, weak extensions, and minimal closed sets for nonmonotonic rule systems.

We acknowledge helpful conversations and discussions with Krzysztof Apt, Howard Blair, Michael Gelfond, Winniefried Just, John Schlipf, V.S. Subrahmanian, and Miroslaw Truszczyński. Special thanks are for John Crossley who read the manuscript carefully and pointed numerous mistakes and possible improvements.

\section{References}

[Apt, 1988] K.R. Apt. Introduction to Logic Programming. TR-87-35, University of Texas, 1988.

[Apt, Blair and Walker, 1987] K.R. Apt, H.A. Blair, A. Walker. Towards a theory of declarative knowledge. In: J. Minker ed. Foundations of Deductive Databases and Logic Programming, pp. 89-142, Morgan Kaufmann, Los Altos, CA.

[Bidoit and Froidevaux, 1988] N. Bidoit, C. Froidevaux. General logical databases and programs, default logic semantics, and stratification. J. Information and Comput., to appear.

[Blair, Brown, and Subrahmanian, 1989] H.A. Blair, A.L. Brown, V.S. Subrahmanian. Monotone Logic Programming. Technical Report CS-TR-2375, University of Maryland.

[de Kleer, 1986] J. de Kleer. An Assumption-based TMS. Artificial Intelligence 28:127 - 162, 1986 . 
[Dilworth, 1950] R.P. Dilworth. A decomposition theorem for partially ordered sets. Annals of Mathematics 51 (1950) pp. 161-165.

[Doyle, 1979] J. Doyle. A Truth Maintenance System. Artificial Intelligence Journal 12:231-272, 1979.

[Gelfond and Lifschitz, 1988] M. Gelfond, V. Lifschitz. Stable Semantics for Logic Programs. In: Proceedings of 5th International Symposium Conference on Logic Programming, Seattle, 1988.

[Gelfond and Lifschitz, 1989] M. Gelfond, V. Lifschitz. Logic Programming with Classical Negation. Unpublished Manuscript.

[Gelfond and Przymusińska, 1986] M. Gelfond, H. Przymusińska. On the Relationship between Circumscription and Autoepistemic Logic. In: Proceedings of the ISMIS Conference, 1986.

[Gelfond and Przymusińska, 1989] M. Gelfond, H. Przymusińska. Inheritance Reasoning in Autoepistemic Logic, Manuscript, 1989.

[Gries, 1981] D. Gries. The Science of Programming. Springer-Verlag 1981.

[Philip Hall, 1935] P. Hall On representatives of subsets. Journal of London Mathematical Society 10 (1935) pp. 26-30.

[Marshall Hall, 1948] M. Hall. Distinct representatives of subsets. Bulletin of American Mathematical Society 54 (1948), pp. 922-926.

[Halpern and Moses, 1984] J.Y. Halpern, Y.O. Moses, Knowledge and Common Knowledge in a Distributed Environment, 3rd ACM Conference on the Principles of Distributed Computing, pp. 50-61.

[Hintikka, 1962] J. Hintikka Knowledge and Belief. Cornell University Press.

[Huang and Nerode, 1985] W-Q. Huang, A. Nerode. Applications of Pure Recursion Theory to Recursive Analysis. Acta Sinica 28.

[Konolige, 1988] K. Konolige. On the Relation between Default and Autoepistemic Logic. Artificial Intelligence 35:343-382, 1988.

[Marek and Nerode, 1990] W. Marek, A. Nerode. Decision procedure for default logic Mathematical Sciences Institute Reports, Cornell University.

[Marek and Truszczyński, 1989] W. Marek and M. Truszczyński. Relating Autoepistemic and Default Logics. In: Principles of Knowledge Representation and Reasoning, Morgan Kaufman, San Mateo, 1989. (Full version available as Technical Report 144-89, Computer Science, University of Kentucky, Lexington, KY 40506-0027, 1989.) 
[Marek and Truszczyński, 1989b] W. Marek and M. Truszczyński. Stable models for logic programs and default logic. In: Proceedings of North American Conference on Logic Programming, MIT Press, 1989. (Full version available as Technical Report, Computer Science Department, University of Kentucky, Lexington, KY 40506-0027, 1989.)

[McCarthy, 1980] J. McCarthy. Circumscription — a form of nonmonotonic reasoning. Artificial Intelligence Journal, 13:27-39, 1980.

[Metakides and Nerode, 1977] G. Metakides, A. Nerode. Effective Content of Field Theory. Annals of Mathematical Logic, 17:289-320.

[Minsky, 1975] M. Minsky. A framework for representing knowledge. In: The Pychology of Computer Vision, pp.211-272. McGrow Hill.

[Mirsky, 1971] L. Mirsky. Transversal Theory. Academic Press, New York.

[Moore, 1985] R.C. Moore. Semantical Considerations on Non-Monotonic Logic. Artificial Intelligence, 25:75-94, 1985.

[Nerode and Remmel, 1985] A. Nerode, J.B. Remmel. A Survey of r.e. Substructures. Proc. Symp. Math. 42, Amer. Math. Soc.

[Nerode and Remmel, 1987] A. Nerode, J.B. Remmel. Complexity-theoretic Algebra I: Vector Spaces over Finite Fields. In: Structures in Complexity. pp. 218-241.

[Nerode and Remmel, 1989] A. Nerode, J.B. Remmel. Complexity-theoretic Algebra II: Boolean Algebras. Annals of Pure and Applied Logic 44:71-99.

[Nerode and Remmel, 1990] A. Nerode, J.B. Remmel. Complexity-theoretic Algebra III: Bases of Vector Spaces. In: Feasible Mathematics, Springer Verlag.

[Reinfrank and Dressler, 1989] M. Reinfrank, O. Dressler. On the Relation between Truth Maintenance and Non-Monotonic Logics. In: Proceedings of International Joint Conference on Artificial Iintelligence, 1989.

[Reiter, 1980] R. Reiter. A Logic for Default Reasoning. Artificial Intelligence, 13:81$132,1980$.

[Remmel, 1989] J.B. Remmel. Recursive Boolean Algebras. In: Handbook of Boolean Algebras

[Rogers, 1967] H. Rogers, Jr. Theory of Recursive Functions and Effective Computability McGraw-Hill, New York.

[Tarski, 1956] A. Tarski. Logic, Semantics, Metamathematics Oxford, 1956. 